\title{
Style based automated graphic layouts
}

\author{
Paul Cleveland, Queensland College of Art, South Bank Campus, Griffith \\ University, South Bank, Queensland 4101, Australia
}

This article describes a methodology for producing style based generative layouts which could be applied to magazine or web design. Many graphic designers are passionate about their design work, but mundane and routine design considerations are often left to those less experienced. This paper examines a methodology that can generatively reproduce variations to a design specification based on preset inputs which offer variety in layout without the loss of design aesthetics. An historical survey examines symbolic artefacts constructed from the conventions of a particular culture over time and illustrates how these influence the outcomes of design specifications. A research methodology is used to convert these specifications into generative designs using a set of algorithms.

(C) 2009 Elsevier Ltd. All rights reserved.

Keywords: generative design, design automation, graphic design, style, aesthetics

\section{Corresponding author:} Paul Cleveland p.cleveland@griffith. edu.au
$\mathrm{T}$ The arrangement of letters on a page has been described by writer, typographer, and journal publisher Blackwell (2004) as 'an arcane corner of interest', yet the increased interest in research into visual grammar shows that layout is becoming an important aspect of communication which informs language. Kress and Van Leeuwen $(1996,2002)$ provided a methodology for analysing colour and layout which complemented the language structure, and Van Leeuwen (2006) provided a discourse on the semiotics of typography which alludes to the semiotics of layout. This paper takes this as a starting point and investigates how design specifications for layout can be automated without the loss of design aesthetics.

Previous research into automated layout systems follows two methodologies. The first applies constraints to the layout so as to control the placement of components (Borning and Duisberg, 1986; Vander Zanden and Myers, 1990; Lok et al., 2004). The characteristic solution uses the concept of a grid which has been adapted from traditional graphic design approach to print layout. The other approach uses a set of templates as exemplars which have been produced by layout experts (Myers et al., 1993; Jacobs et al., 2004).

To a large extent layout can be considered formulaic. The development of 'house styles' in magazine publication is an example of how the graphic treat-

www.elsevier.com/locate/destud 0142-694X \$ - see front matter Design Studies 31 (2010) 3-25 doi:10.1016/j.destud.2009.06.003 
ment of space can value add to the branding of an entity. Once a page layout has been considered the implementation often becomes to a large extent a mechanical proposition. There are two distinct parts to this process, firstly the aesthetic skill of arriving at a design solution, and secondly the mechanical skill of repeated implementation with a degree of variation.

The design specification is often a negotiated dialogue between a number of parties which can include the designer, client, marketing professionals, product researchers and consumers. Magazine publishers will rely not only on the written content and graphic imagery but also the arrangement of these elements taking into account such things as colour, proportion, dynamics and scale to attract an audience and bring about loyalty in readership. The design of the layout goes further than providing a framework for functionalism. The design of the pages provides a medium for graphic experiment within the bounds of editorial responsibility. Designers often act as jugglers of creative solutions as well as arbiters of communication format. The influences of subcultures can be a powerful force in the selection of appropriate graphics and typographic arrangement. The use of the same repeated solutions leads to over familiarisation with the content and a diminished stimulation. Designers are therefore always searching for ways to enrich the novelty value of the visual stimulus. This can become a time intensive activity as different variations to a design specification are explored. Often less experienced designers are given the task to fulfil this activity. This research explores the possibility of using a set of generative algorithms to produce variations to a design specification to speed up the layout process.

This research looks at the shape of elements and their placement in a layout, relative to other elements, and how they form a rhetoric based on their characteristics which determines a design style. More importantly the development of style in contemporary communication design can be regarded as complementary to the complex communication derived from the visual grammar, and are as Scott (1994) states 'not merely analogues to visual perception but symbolic artefacts constructed from the conventions of a particular culture'. These conventions of a particular culture influence the outcomes of design specifications. It therefore follows that temporal based design specifications can be reconstructed using rules which govern this visual language. Style can be interpreted as specific combinations of relationships between elements in a particular visual language. The application of this relationship is explored by constructing a set of rules and then applying them to a computer layout system which can generate multiple solutions based on a predefined logic. This is the basis of our generative system. One particular style genre, 1950s is used to test the validity of the logic employed in the development of an automated system to construct variations to a design specification. The decision to use this particular period was based on the need to work with a limited number of variables to see if a set of particular rules could achieve an intended outcome. 
While it is acknowledged that these layouts offer a tighter structure than many contemporary design specifications the application of the principals involved in building the design variations can be translated to more contemporary visual forms.

The intrinsic nature of the shapes which represents the layout elements, their placement and interrelationship construct a context which relates to an historical design period. If layouts were examined from any particular period of the $20^{\text {th }}$ century one could identify particular collective characteristics of shape, colour, weight, scale, orientation, space and relationship which would be unique to that period. Figure 1 is put forward as an example. It shows examples of 1950s layouts for an auto manufacturer. A survey of the elements which make up the layout composition and their relationship to one another and other elements can give one a formula for constructing other variations. By recognising the formulaic structures of any time period pastiches can be constructed which to a large extent represent those time periods.
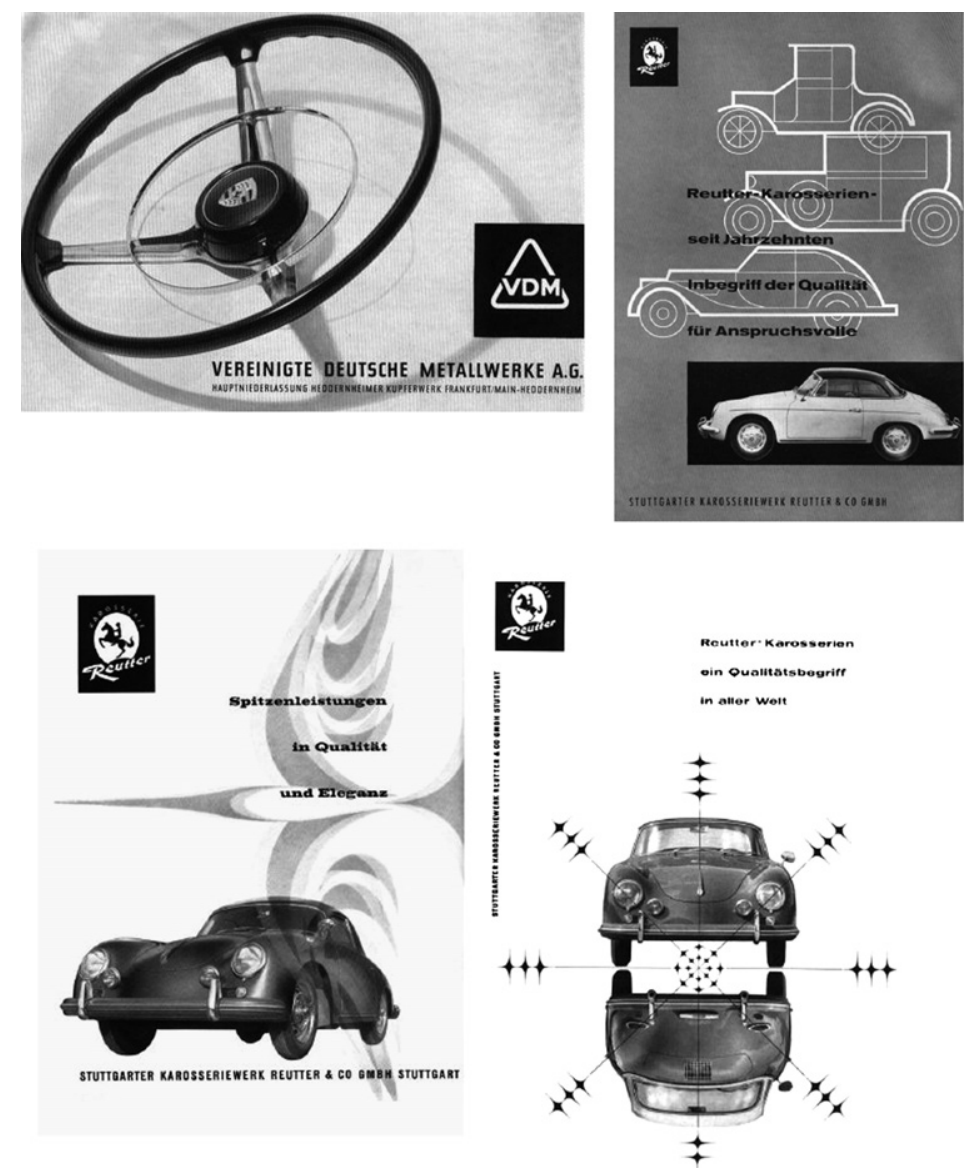

Figure 1 German auto ads from the 1950 s

Style based automated graphic layouts 
Figure 2 takes the same photographic elements as appeared in Figure 1 and reinterprets the layout using collective relationships typical of the 1960s (A), the 1970s (B), the 1980s (C), the 1990s (D), and the turn of the $21^{\text {st }}$ century (E). This exercise illustrates that it is possible by manipulating a number of variables to come up with differing stylistic results.

\section{Stylistic attribution}

In any page layout system the complex arrangement of various graphical elements builds the visual language. This language has its own syntax which determines the reading of the elements in terms of saliency (that is the order of importance) and in turn derives meaning from a particular visual culture. Any arrangement of particular elements in a predefined way will lead to a grouping of like outcomes which can be regarded as a style. Typically journals from particular time periods tend to produce a house style which in many cases becomes the influence of other house styles, thus leading to period styles (Cleveland, 2004). The author has devised a methodology for tracking the relationships between elements which is known as a Style Attribution Index (SAI). This index consists of form elements, saliency relationships and treatments which all go to build a style ontology. The use of ontology here is in the sense of a data model which represents the relationships which exist between the different entities.

Perhaps the most important question to this endeavour is its end purpose. Many of the tasks associated with design layout are repetitive and follow predefined rules. Automation of these tasks would free up designers to take on more creative work.

Large commercial publishers are rarely the innovators of design styles. Innovation is a risky business and radical changes in the visual language of a publication may experience demographic shifts to, or away from readership. Therefore, the changes of style over time tend to be gradual and based on the prevailing communication technologies of the time. Innovation tends to come from niche brand publications such as Wired, Colours, I-D and The Face.

Form elements are the rudiments of layout composition. The tracking of such elements over time illustrates the fluid nature of style change. This phenomenon can be seen in the cover designs of Vogue magazines from 1916 to the present day (Figure 3).

The 1916 design is text salient, which is a manifestation of the prevailing text biased visual grammar of the era. The illustrative material becomes a secondary consideration. By 1919 the prominence of text elements is reversed to a domination of illustrative material. During this period, illustrators such as Christian Berard and Georges Lepape developed an illustrative style which became synonymous with elegance and exclusivity. During the late 1920s and 

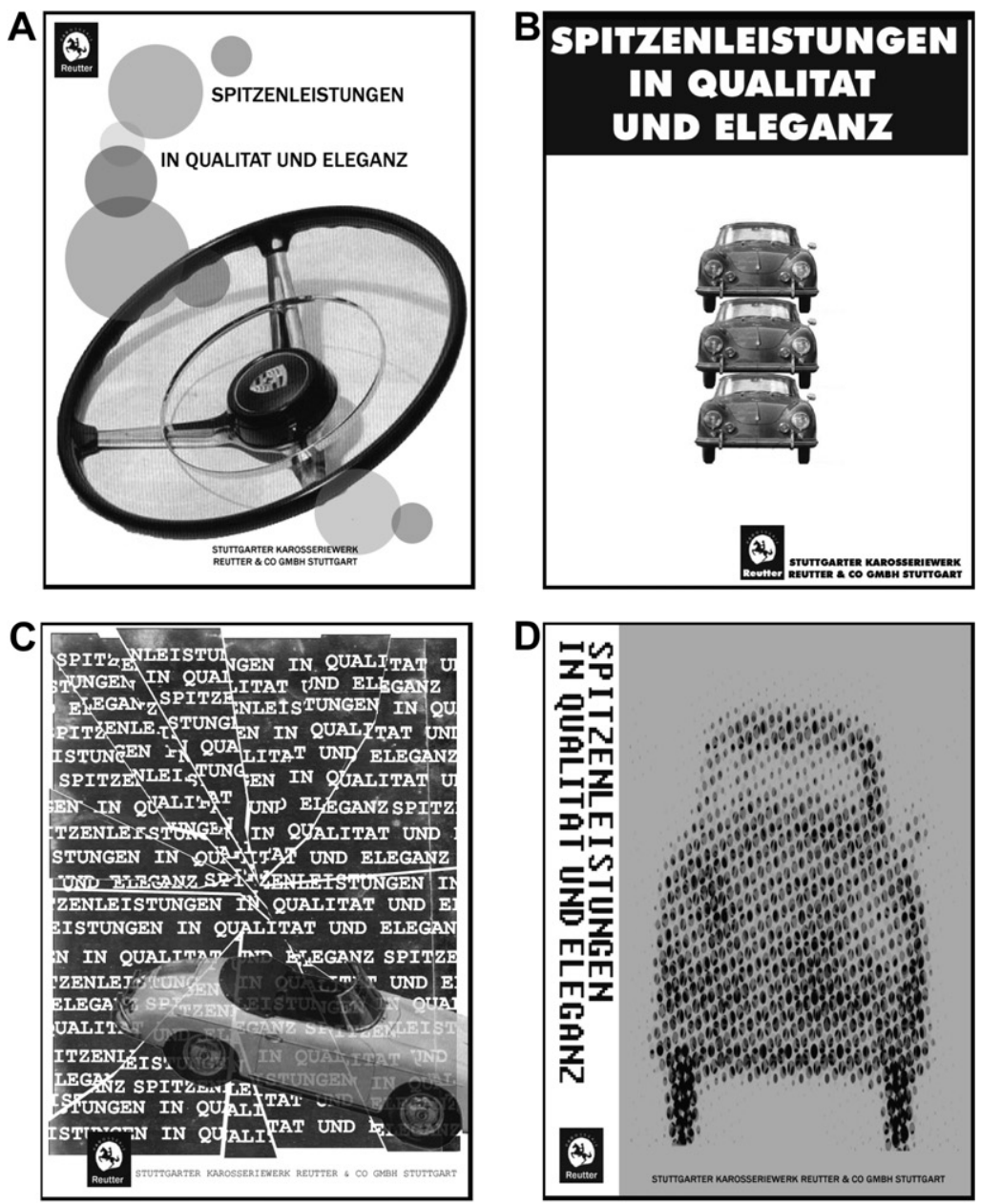

Figure 2 The use of collective

$\mathrm{E}$

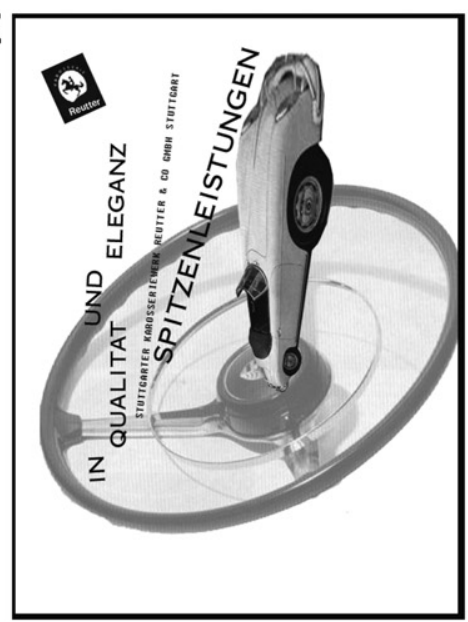

layouts 


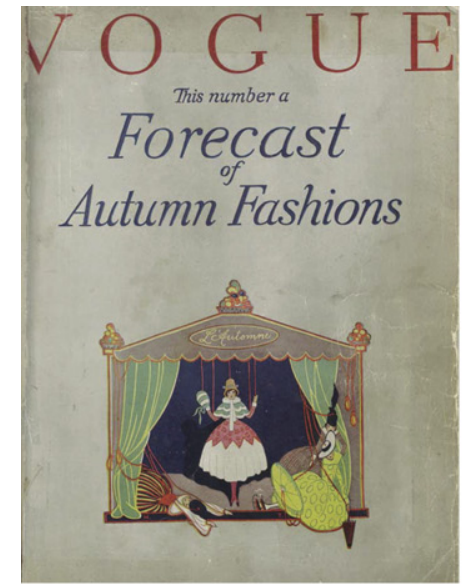

1916

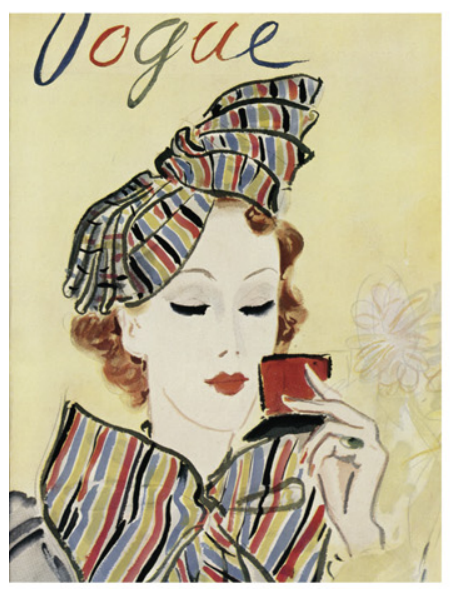

1935

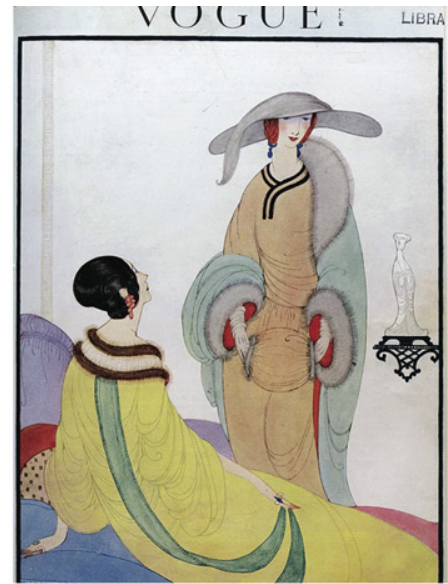

1919

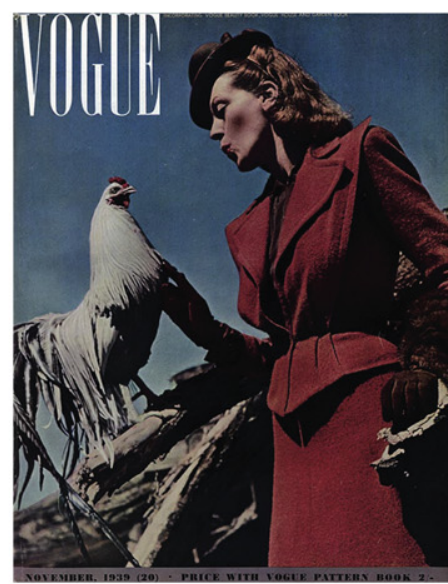

1939

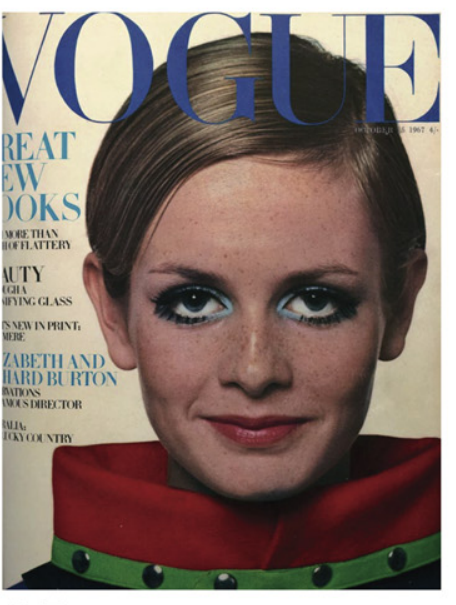

1967

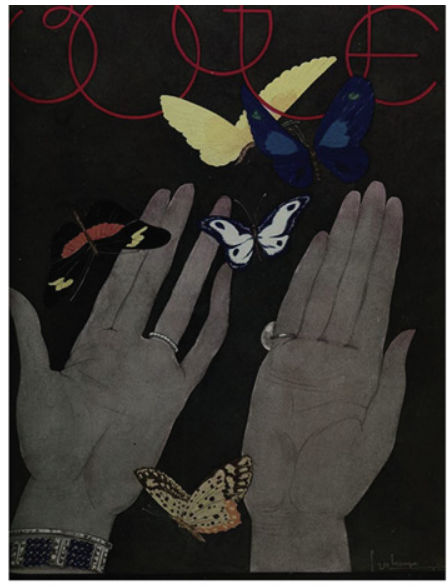

1931

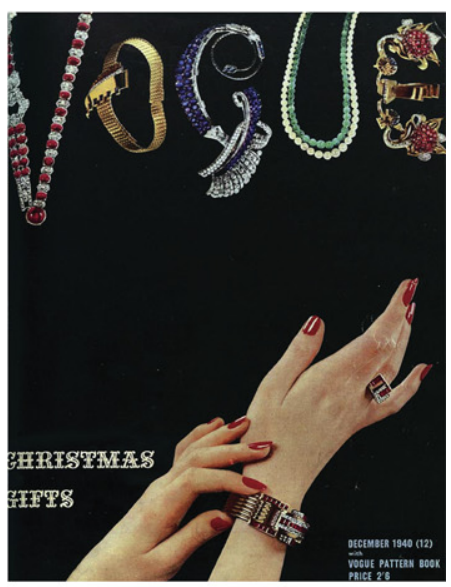

1940

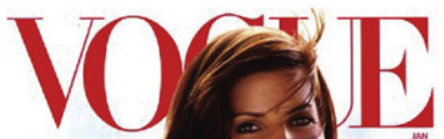

Sandra

Bullock

Takes

Manhat

NEW HAR,
NEW CLOIHES

\&ARONTRO

time for

change

TH'

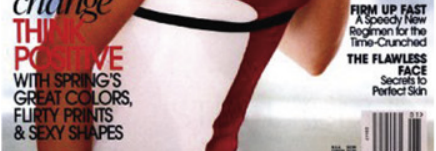

2006

Figure 3 Vogue covers 1916-2006 
early 1930s the masthead logotype, which was based on geometric shapes changed with each edition. The illustrative technique of this period was predominated by the use of flat colour, but there were already examples of photography appearing in layouts. These images were influenced by the movie industry and the art movements of the time. By the mid-1930s the cover art had changed to a loose painterly technique. The masthead also took on this appearance. Photography made its cover debut in 1936 and has dominated ever since. One could argue that this was a direct result of technological determinism, and we see that the use of photography was to have a powerful influence on the subject matter for the designs in the post 1950s years.

The war years of 1940-1943 saw the domination of photography as the preferred medium. This even influenced the design of the masthead. The first appearance of the distinctive sans serif masthead which is used today was in 1939, albeit in a rather compact version. By 1940 this was to become the masthead design of choice. Post war marketing had established that the value of brand resided in the identification of a particular logotype. This required the exploration of other avenues to heighten and hold the viewer's interest, this is a term known as the arousal potential.

Yarbus (1967) had shown that people's faces were consistently shown to be the most salient parts of an image. Cerf et al. (2007) note that 'observers even when not instructed to look for anything particular, fixate on a face.' From the early 1960s up until the present day, the notion of the subject looking at the viewer of the magazine has become the dominant design model. From 1980 up until the present just about every Vogue cover employed this solution. The human attribution of making eye contact is a powerful attraction mechanism, but there are other methods which were used to increase arousal.

From the late 1940s text content teasers were used as an arousal device on magazine covers. By the 1960s this design feature had increasingly taken over the real estate of the magazine cover. To heighten the impact the original light serif type was changed to bold italic sans serif. This can be seen in a comparison of the 1967 cover to that of 2006. By the 1990s not only had the number of text items increased, but also the point sizes became larger and lowercase was used as it tended to let the background image show through the glyph negative spaces more than the upper case characters. The 2006 cover in Figure 3 shows the further adaptation of text related features to enhance the arousal potential. Here we have not only all caps statements in various point sizes, but also caps and lowercase in a number of contrasting fonts and colours. The complexity of the placement and variation of elements have increased the arousal potential of such designs. To illustrate that this development of visual language was not an isolated circumstance of Vogue house style samples are provided for Cosmopolitan magazine which reflect a similar style development (Figure 4). 

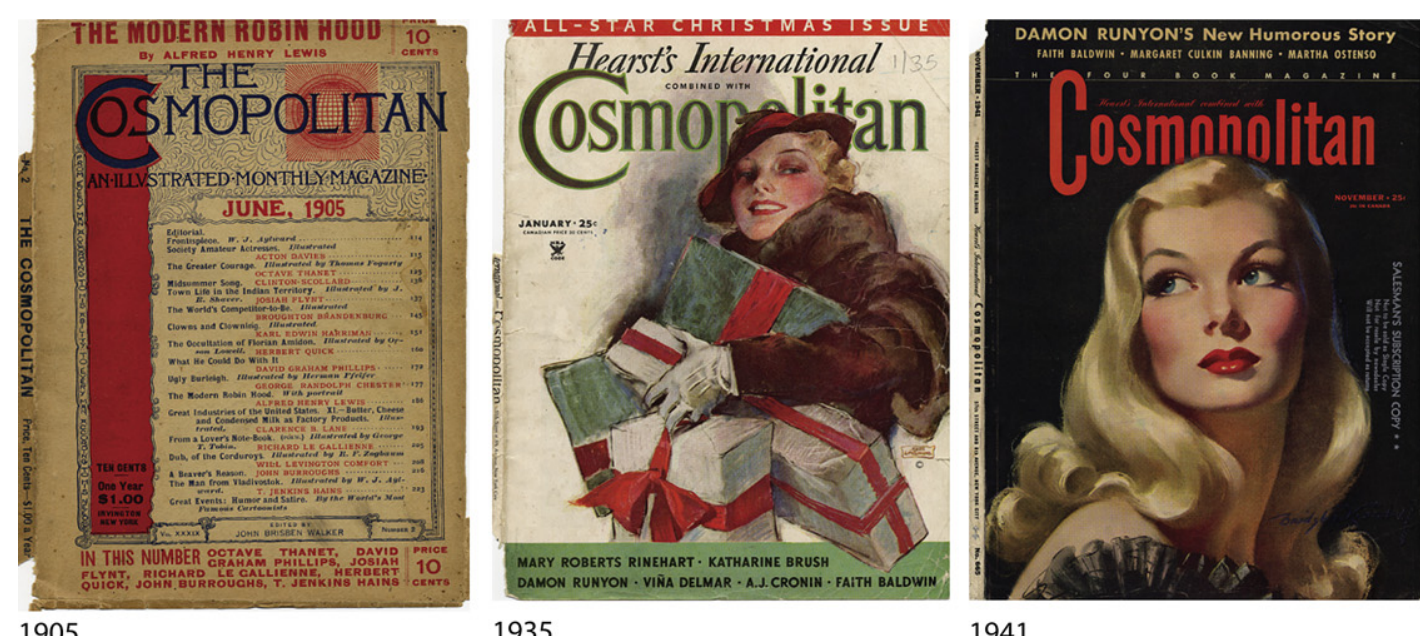
1935

1941
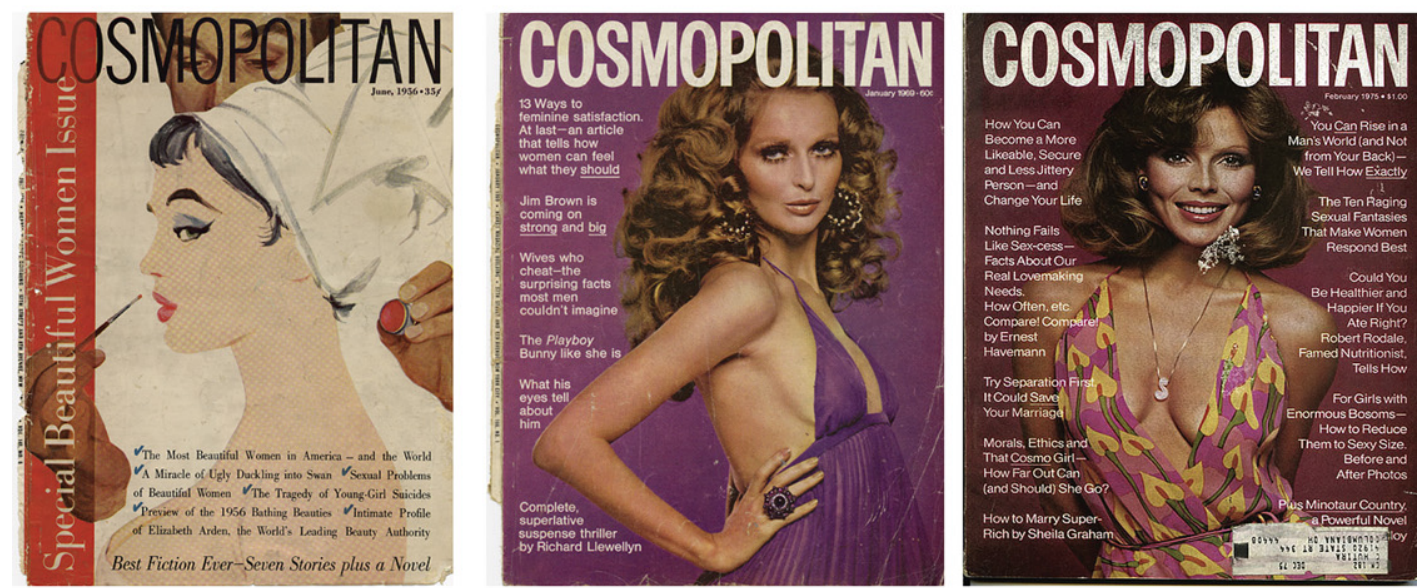

1956

1960

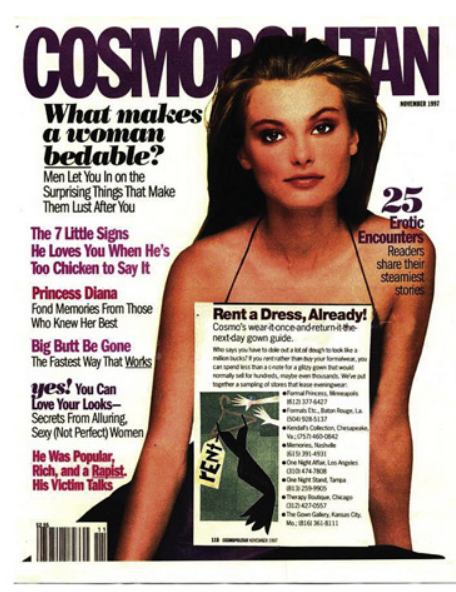

1997

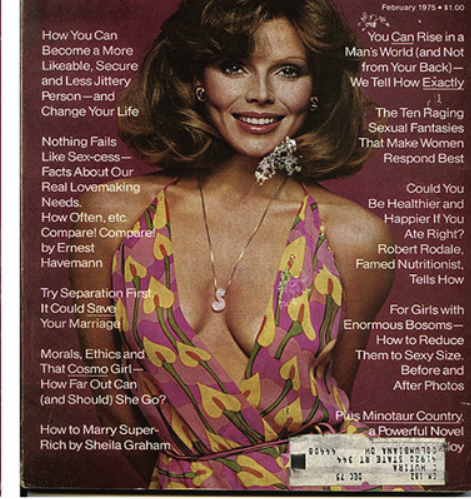

1975

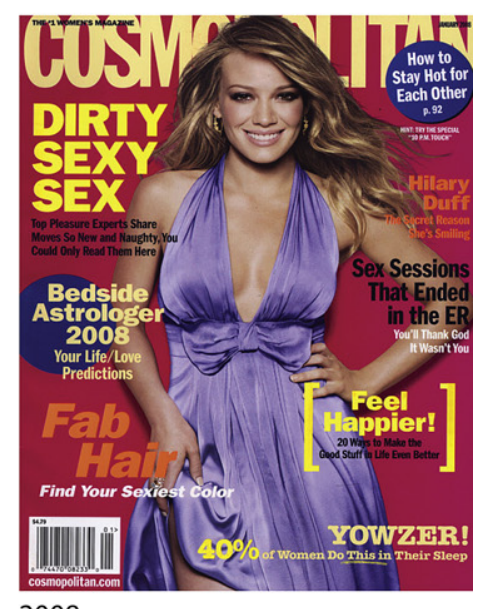$$
1997
$$

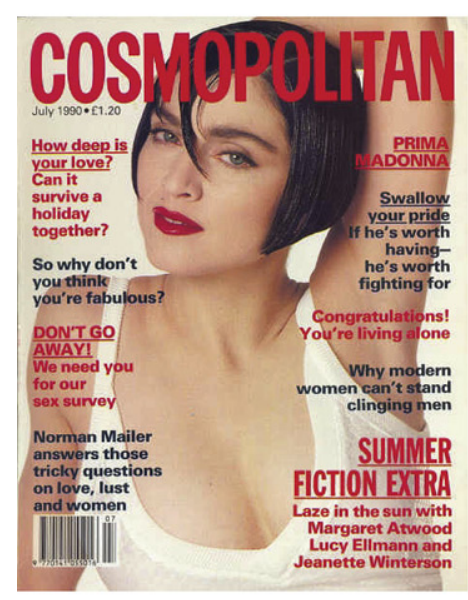

Figure 4 Cosmopolitan covers 1905-2008 
We can see from the different Vogue/Cosmopolitan examples that different historical eras have their own stylistic attributions which make up the visual grammar of the day. These are found in the elements which make up the design; graphic devices, text, illustrations and photographs, as well as the proximity of placement. On another level the inscription characteristics of colour and patterning are also important devices for establishing stylistic attributes. By varying these attributes any design solution from one style can be converted to another. In doing this it should be recognised that the communication relationship also changes. One could argue that there is a kind of Sapir-Whorf (Kay and Kempton, 1984) effect at work. The reading of a design layout outside its origin time would be considerably different from that of a contemporary viewer. Therefore, it could be argued that the mindset of a 1950s designer would be completely different from that of a contemporary designer. What may have appeared to have high values of arousal in the 1950s may, or may not have the same value today. The retro fitting of stylistic variables in a contemporary period, which is evident in the period post 2000, shows that style based design can still have a powerful arousal potential.

\section{Construction of the Style Attribution Index}

To be able to reproduce stylistic variations in a layout we need a mechanism which can offer meaningful variables from which we can make choices. The Style Attribution Index (SAI) is a logic system which allows such choices. The SAI first of all consists of a series of elements which form a layout. The first consideration is the saliency of the elements which make up the layout. The order of saliency is an important aspect in building a style attribution index as it establishes relationships between the elements.

Figure 5 illustrates a number of ways in which the saliency can be read. The solution on the left in Figure 5A indicates the saliency using Yarbus' research findings. It is based on a top down attentional bias which attempts to make sense of the elements. Therefore, the eyes of the large face become the most salient followed by other faces and then the headline and bodytext.

In the 1953 layout on the top right (B) a set of bottom-up attention neuromorphic vision algorithms developed by iLab at the University of Southern California (Lee et al., 1999) are used to identify the saliency path. This set of algorithms has been shown to be robust and close to primate responses. A detailed explanation of the process can be found in 'Computational Modelling of Visual Attention' (Itti and Koch, 2001). The result on the right is an anti clockwise eye circulation which is based on visual attributes such as intensity, contrast, colour opponency, orientation and direction (Itti and Koch, 2001) without making any sense of the imagery. It is beyond the scope of this paper to examine these attributes but Itti and Koch (2001) paper gives a comprehensive overview of the process. Saliency in this case is based on colour shape, 
A

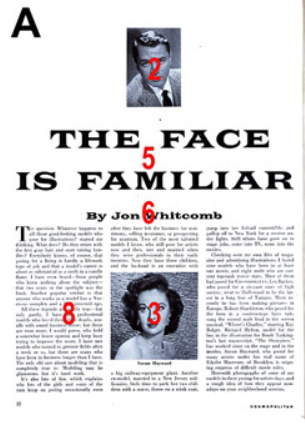

C

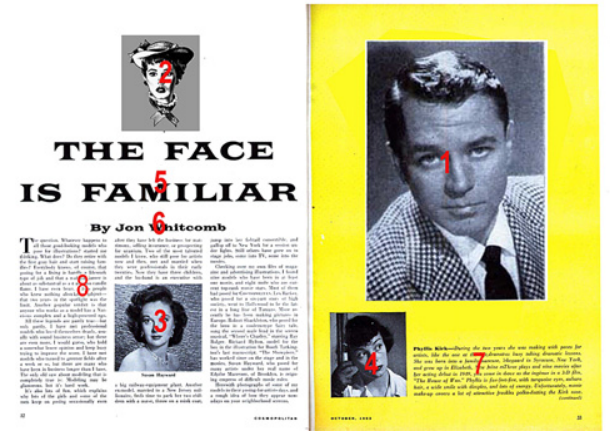

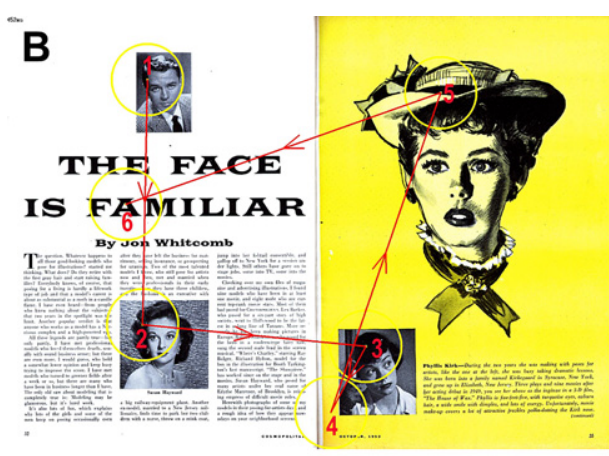

D

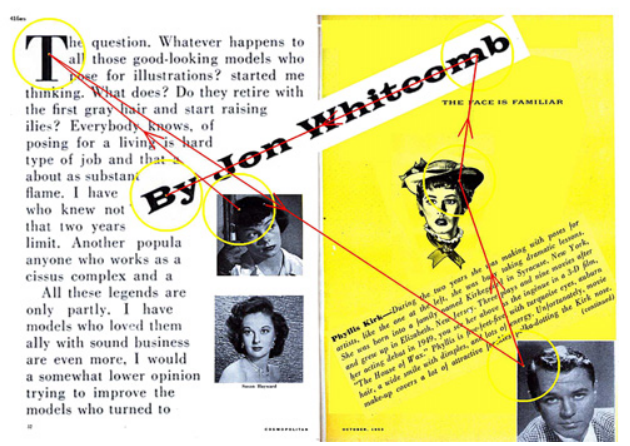

Figure 51953 Cosmopolitan layout, left - top down solution, right - bottom-up solution

form and placement without any reading of the content. It has value as a diagnostic tool.

The order of saliency for the photographs need not be made explicit by the designer, and in any design variation they could be interchanged as can be seen by example $5 \mathrm{C}$. This is not the case with text as typographic conventions associated with layout dictate to some extent the saliency order, example 5D illustrates a change in the text saliency order thereby changing the style of the composition. As these are double page examples and convention reads from left to right and from top to bottom, the directional bias of saliency is usually in the anti clockwise direction, even to a large extent in example 5D.

Taking Kress and Van Leeuwen's (2001) concept of type being a semiotic resource which is organised as a mode, the features of the text elements in particular combinations further define the building of style by their saliency relationship. On the surface, layouts such as those in Figure 5 seem to be quite simple, and to an experienced designer not overly complex. In reality the judgements associated with the components of the SAI such as symmetry, balance and equilibrium are complex when the elements in a layout are analysed. The SAI contains the parameters which define the scope of choices which can be made. These can be illustrated as a semantic differential where choices can 
be made on appropriate settings (see Figure 6). This represents the first layer of the SAI where presets can be made and choices identified.

\section{Development of a grid}

The placement of elements is determined by an aesthetic function which determines the order and complexity of the designs. The computational formula used in this solution is based on the work of Birkhoff. Birkhoff (1933) produced a formula $\mathrm{M}=\mathrm{O} / \mathrm{C}$ which produced an aesthetic measure for a polygonal shape. The measure (M) was equal to order $(\mathrm{O})$ divided by complexity $(\mathrm{C})$. The sum of the vectors which describes a shape became the measure for complexity. This same idea can be applied to layout designs to produce stylistic variables which allow variation of complexity in a design. Complexity can be seen as an arousal creating mechanism similar in concept to the use of complexity in the latter designs of the Vogue covers. This can also work in an inverse way where order would be an arousal inhibitor.

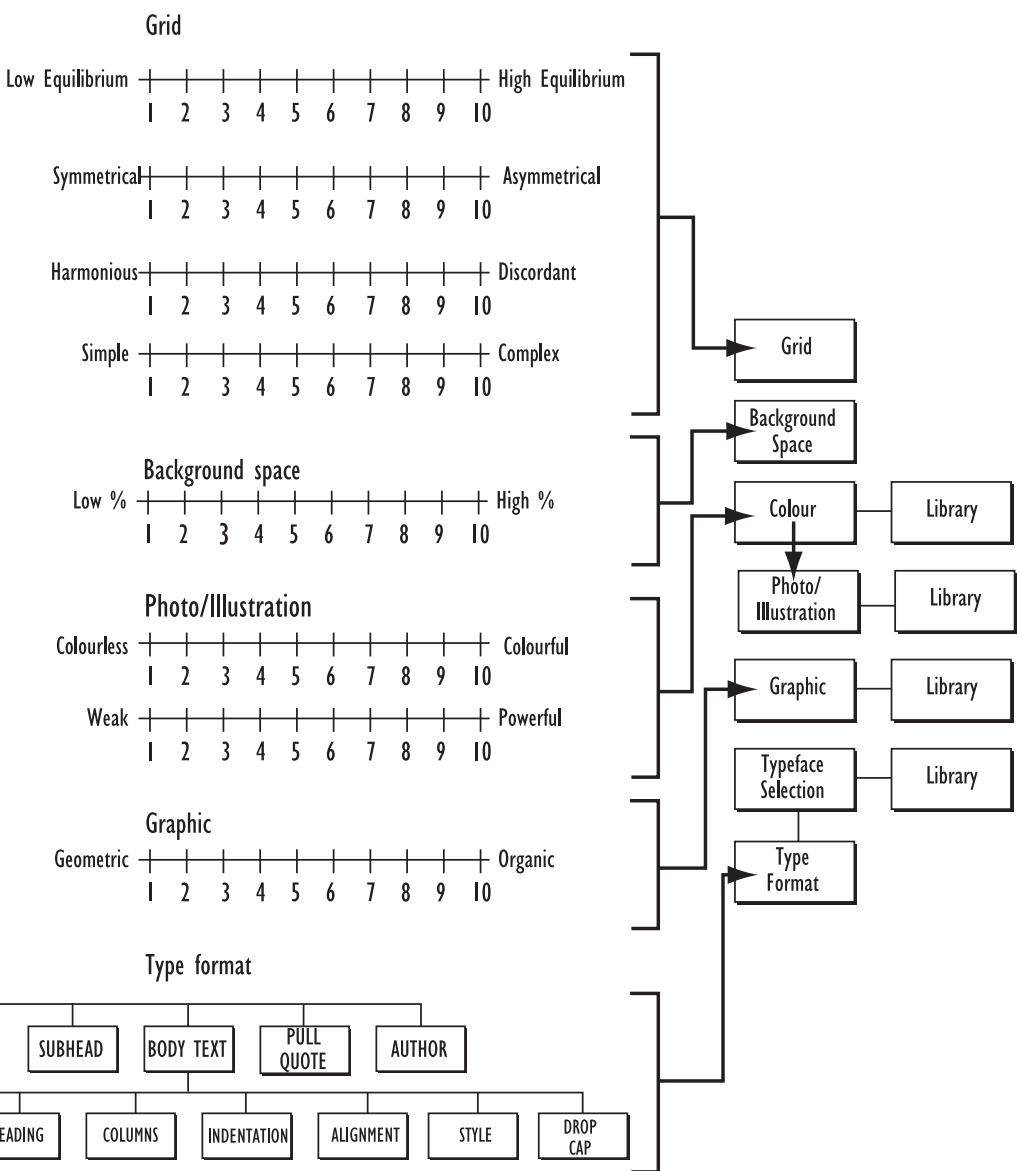

Figure 6 Semantic differential settings and logic saliency of the Style Attribution Index

Style based automated graphic layouts 
The example in Figure 7 illustrates how this is applied to a generative layout process. The complexity is calculated on the least number of extended horizontal $(H)$ and vertical $(V)$ gridlines it would take to produce the layout. Added to this is the number of different elements $(E)$ in the layout. The formula being $C=\sum_{i=1}^{n} H+\sum_{j=1}^{n} V+\sum_{k=1}^{n} E$.

The grids in Figure 7 use high values of symmetry in their construction but show differences in complexity which correspond to the differences in layout solutions. The layout on the left has a complexity value of $16(5+5+6)$ which can be seen as a result of a symmetrical grid of even distribution. The other two examples use the same elements but have differing values of complexity. The grids are still symmetrical but they are based on the golden section (an aesthetic ratio of 1.618) rather than evenly spaced. Their complexity values are for the middle $24(9+9+6)$ and the right $32(13+13+6)$. Both these solutions show greater values of energy than the solution on the left.

An algorithm was developed to generatively layout the various layout elements through the use of a grid which allowed variations of complexity, symmetry equilibrium and harmony. There are no judgements made on the aesthetic ideals associated with differing values for these parameters. Figure 6 illustrates the four grid parameters. Equilibrium is defined as a condition in which the placement of the mass of each element results in a stable and balanced design. In this experiment the values of equilibrium varied depending on the complexity of the underlying grid system. Symmetrical values determined if gridlines were mirrored across the horizontal/vertical centre of the layout. A harmonious/discordant dimension affected the grid orientation. Low values constrained the grid to horizontal and vertical solutions while high values allowed diagonal lines which range from parallel to random angles. The complexity value determined how many grid options were available,
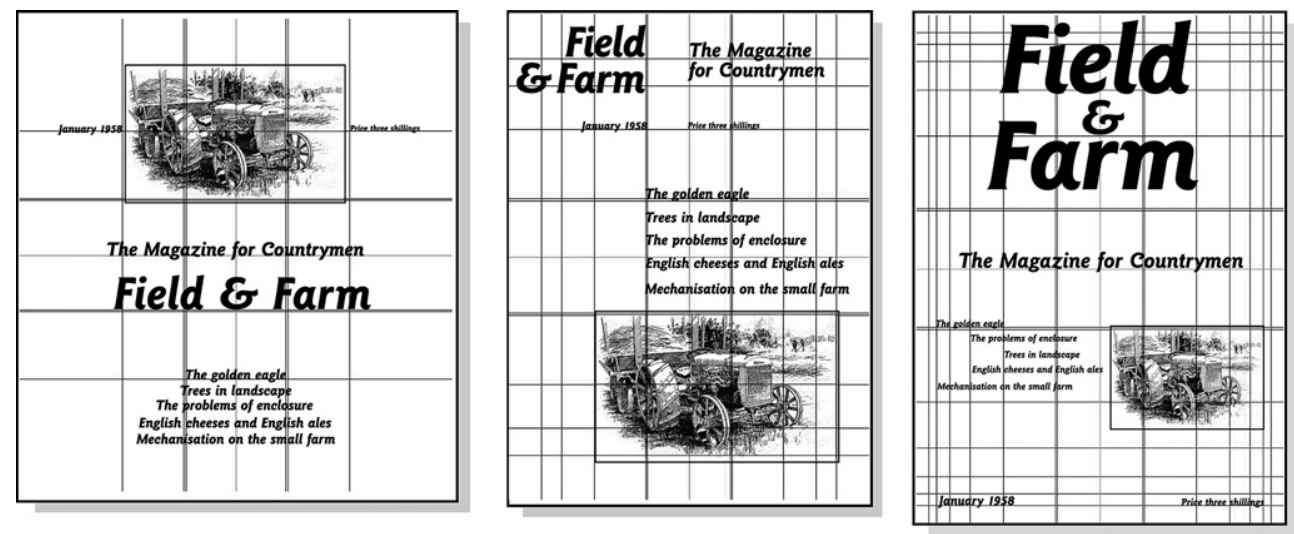

Figure 7 Formation of the grid 
as well as other considerations such as number of typefaces and elements which could be used in any solution.

The generative program was written in 'Processing', a java based visualisation tool. Figure 8 illustrates some of the early layout solutions based on various inputs. These results are not yet mapped to a grid so they show random placements looking for the best solutions associated with the inputs. The image on the left shows high values for horizontal and vertical symmetry, as well as for balance and equilibrium. The image on the right is concerned only with equilibrium so heavy areas of image elements are offset by open space.

Figure 9 illustrates a few of the results of a more robust set of algorithms. The image on the left has low values of complexity and high values of equilibrium. This can be clearly seen from the symmetrical nature of the layout. The algorithm determines the equilibrium point of each object and adjusts the placement accordingly. In the image on the left the large black area of the illustration represented by the hair and hat, is balanced by the white text placed at the lower left.

The algorithm we have developed takes inputs for the number of objects and their relative size. Higher size values allow greater variation in dimensions amongst the elements. Choices can be made as to the inclusion of values for horizontal and vertical symmetry, balance and equilibrium. The Simple/Complex dimension determines the range of grid options available to apply a solution, while Balanced/Unbalanced determines the position of the gridlines. This parameter was an average of the symmetry and equilibrium measure. Low values produce a symmetric and balanced design while high values produce a design which is purposely unbalanced.

The centre image in Figure 9 illustrates a layout with increased complexity. The image on the right illustrates how the layout fits to a relatively more complex grid. The grid structure is a direct result of the values ascribed to
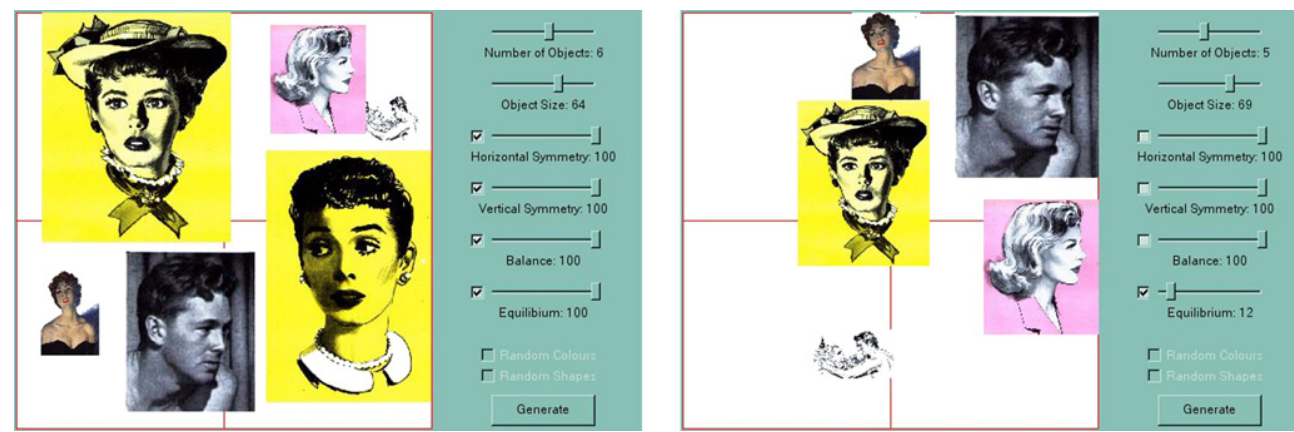

Figure 8 Examples of the placement of elements using the algorithm

Style based automated graphic layouts 

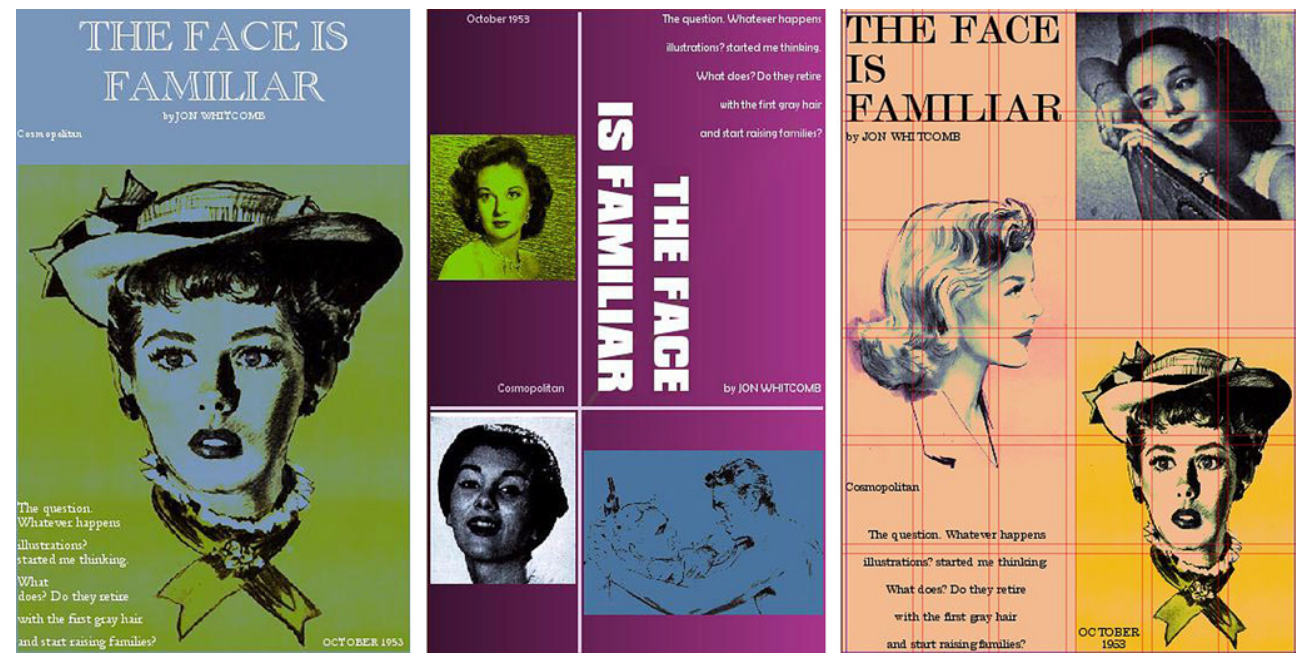

Figure 9 Generative layouts using a 1950s aesthetic

symmetry and equilibrium. There are possibilities for variation to the grid structure based on other aesthetic proportions.

To test the validity of the algorithmic logic a 1950s style genre was chosen as it was felt that the 1950s aesthetic could be easily accommodated using the tools which were developed. The parameters identified in Figure 10, such as a balanced composition without tension, importance of negative space, the use of limited colour pallets and the placement of text and graphic elements to support a balanced composition could all be accommodated using the set of algorithms.

Kadinsky (1979) stated that the aesthetic composition of elements is determined by their relationship to the edge frame. Kadinsky theorised in a subjective manner that as an element approached the edge frame the sense of tension seemed too increased; however, when the element touched the edge the tension dissipated. So to maximise the tension in an image the elements should be placed around the edge of the frame. Typically 1950s layouts do not exhibit high values of tension so centrally placed elements would be an optimum solution. The Weak/Powerful dimension of the Photo/Illustration parameter in Figure 6 catches this phenomenon by allowing overlapping and cropped images. The dimension of Harmonious/Discordant of the grid parameter also effects the orientation of images and text by allowing non-horizontal and vertical grid solutions depending on the values chosen.

Figure 11 illustrates these phenomena as a style variable. Figure 11A illustrates a layout using the set of rules based on a typical 1950s solution. The saliency vectors indicate the way the image is interpreted. This corresponds to Kress and Van Leeuwen's (1996) reading path. Figure 11B is basically the same as 


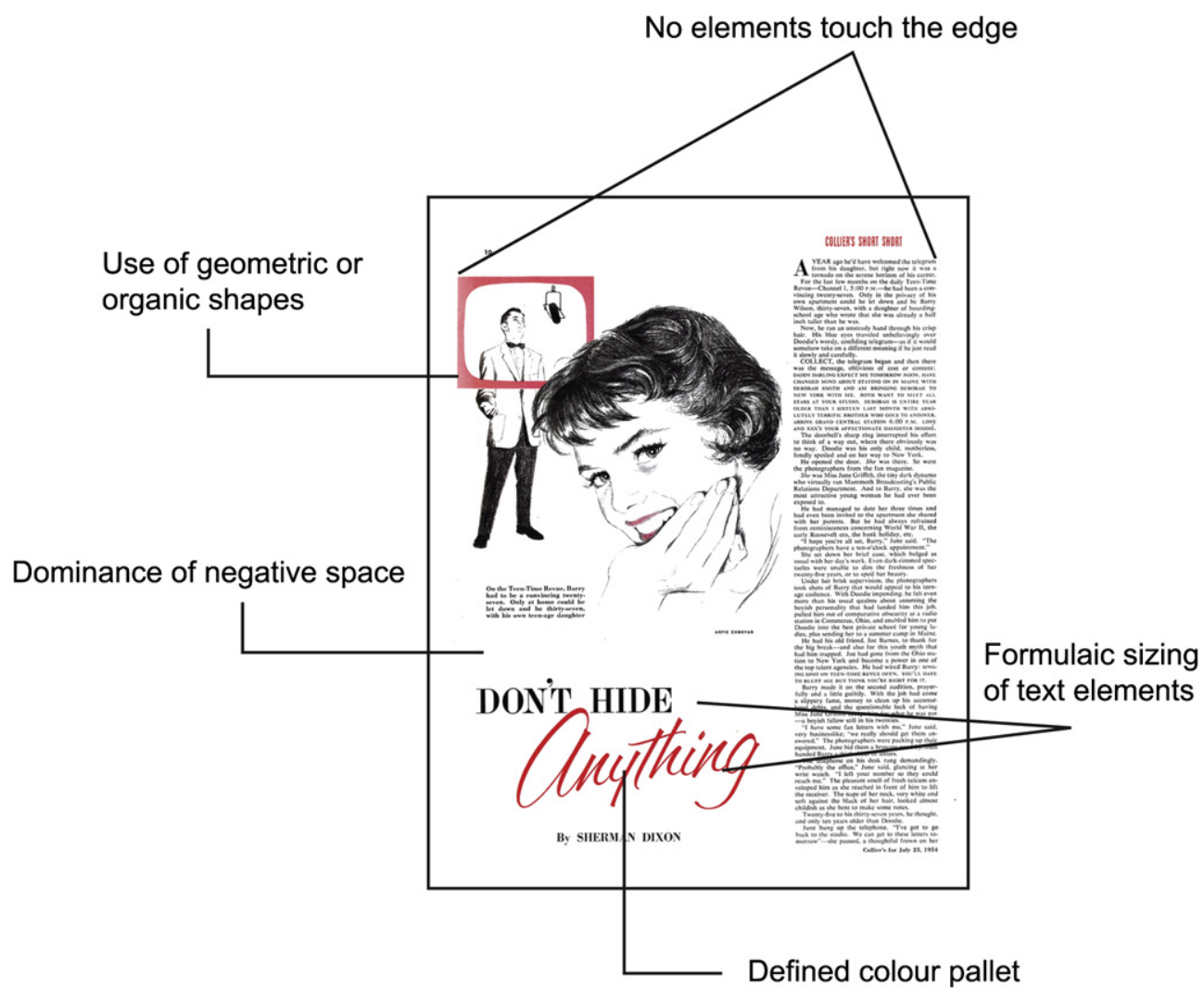

Figure 10 1950s Aesthetic parameters

Figure 11A but without the images touching the edge. It is difficult to see that the tension factor was larger in this layout. In Figure 11C the layout exhibits greater values of tension due to the overlap of images and there is a strong diagonal vector. Figure 11D shows greater tension with low values of equilibrium and symmetry with elements hugging the edges of the layout. The saliency is interpreted as a diagonal. In terms of the SAI we can say that solutions A, B and C are acceptable with variations as a 1950s solution for a particular genre, while $\mathrm{D}$ is not.

\section{Other SAI elements}

So far we have only looked at image placement in relation to other elements within a layout through the construction of the grid. One of the most influential components of style is the use of space with a layout. Space is the real estate in which the elements populate. The amount of information that is placed in a layout is governed by the picture to text ratio and the amount of space that is left vacant. Although space can be termed vacant it still performs a significant influence over the overall design style. The program algorithm can adjust the image and text size to a preset ratio. 

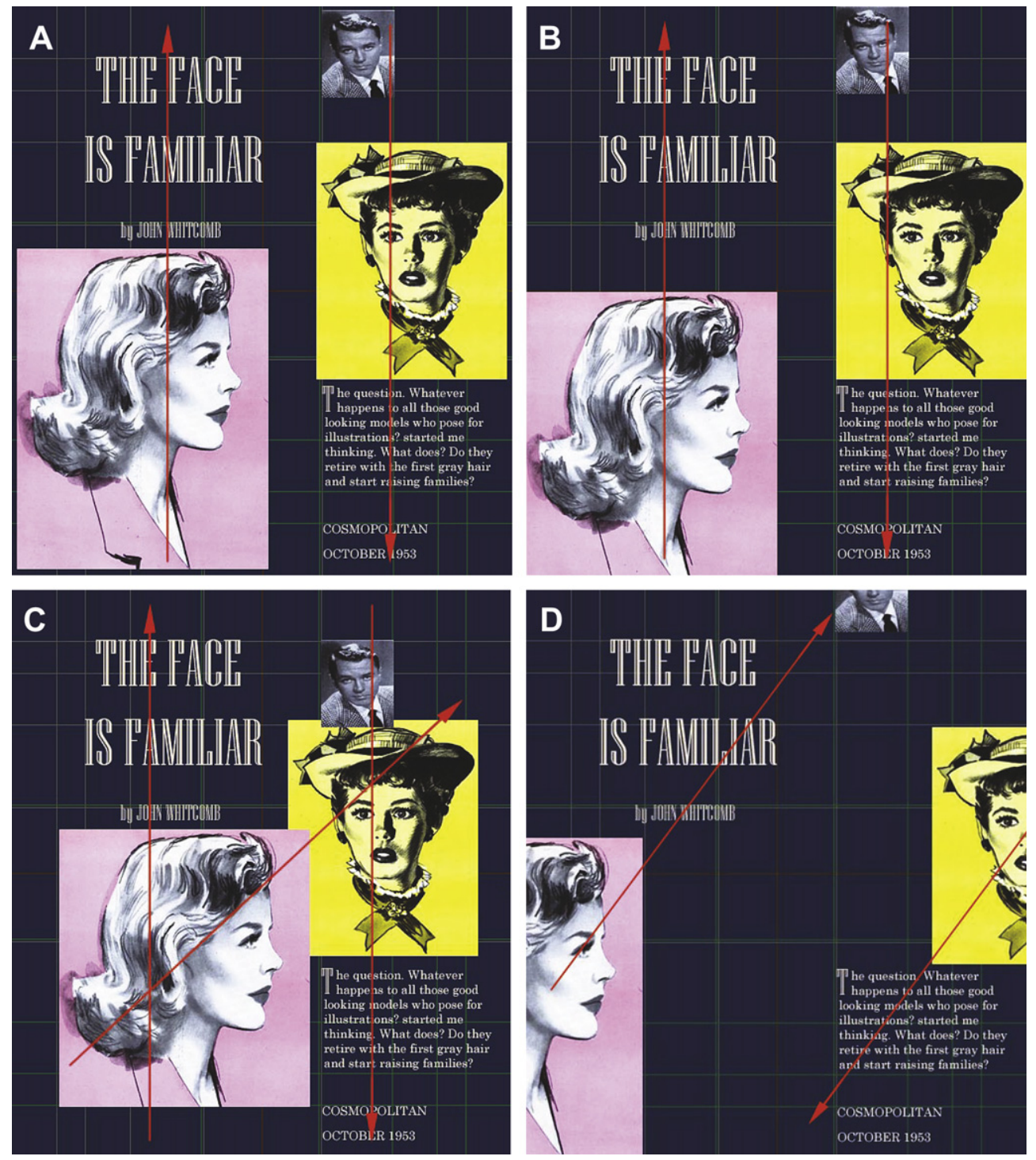

Figure 11 Golden section grid structure indicating saliency vectors. (A) Layout using generative rules, (B) layout with edge tension, (C) layout with greater values of tension due to overlap, (D) greater tension with low values of equilibrium and symmetry

As an example, magazine layout for fiction content from the 1950s shows a distinct use of a formulaic design structure. The elements of these layouts consist of an illustration, headline, subhead, body text, drop-cap, pull-quote, author name, illustrator attribution and running head. As we have seen all these elements determine the saliency, and their relationship to each other to a large extent determines their position in a layout. Although there can be a considerable number of these elements their dominance is not always apparent, and in some cases the negative space tends to dominate. The term negative space refers to those areas of a layout which are not occupied by layout elements. To 
establish if there is a dominance of the elements over negative space a series of 1950s double spreads (1950-1958) from popular fiction magazines were analysed. Comparisons were made initially from eight different publications, and then five spreads from one magazine were analysed in the same manner (Figure 12). This illustrative genre layout solution is based on the ability of the image to be deep etched. Each of the images was converted to greyscale and a threshold. This converted foreground objects to black and background to white. A count was then applied to the pixels, which were then converted to a percentage.

Means for both groups of magazines showed that the percentage of negative space pixels was larger than layout elements (Figure 13). Although there were variations in the counts, to obtain a stylistic impression which replicated those in Figure 12 the ratio of negative space would need to be between 10 and $20 \%$ greater than that occupied by layout elements. Figure 14 illustrates two different solutions using this ratio.

The layout elements are sourced from various libraries depending on which element is to be placed as can be seen in Figure 6. For example, once the number of photographs or illustrations to be included is determined the colourful and powerful parameters determine if the colour depth and saturation of the original image is maintained or decreased. Colour is an important style modifier. Combinations of certain colours can reflect time periods. Low values of colour produce black and white solutions while high values use full colour. Solutions with values close to colourless and weak produce solutions of grey values. The Weak/Powerful dimension also determines if the image is cropped or has the ability to be overlapped with other elements. Figure 15 illustrates the use of predefined colour pallets for 1950s type solutions which are obtained from a predefined library which offers interchangeable options.

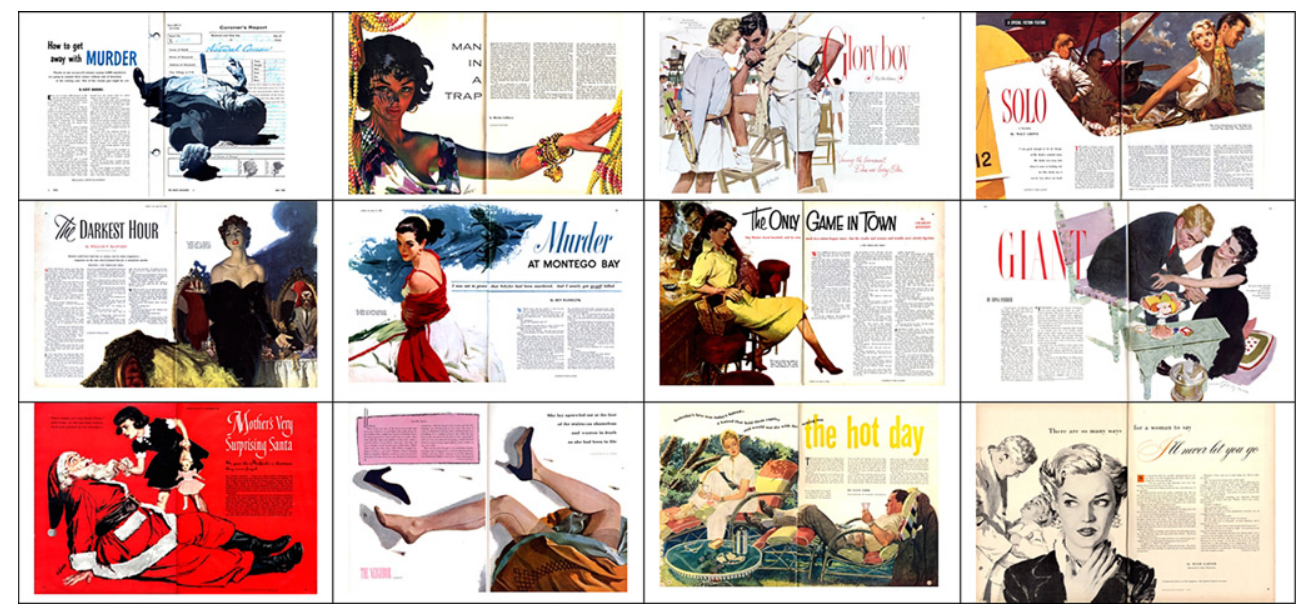

Figure 12 Double spreads used in the background comparison test

Style based automated graphic layouts 

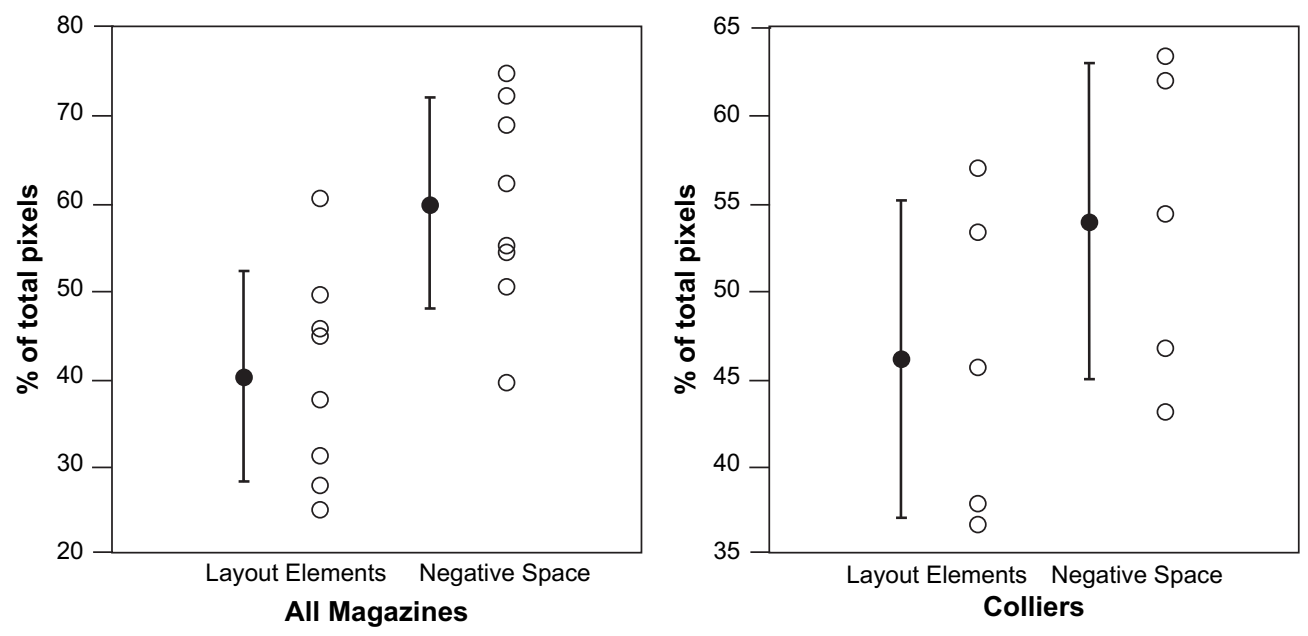

Figure 13 Point and column means for negative space and layout elements

More complex solutions can also include the addition of graphics as part of the design solution. The SAI Graphic parameter of Geometric/Organic refers to generatively produced graphics. Figure 16 illustrates the use of geometric graphics on the left with the use of triangles, and organic objects on the right in the form of blob type shapes in the book title graphic.

Again, a library of solutions is available to construct such solutions. Algorithms generatively construct graphics which can be used as background patterns or linking devices for other elements. Figure 17 illustrates on the left
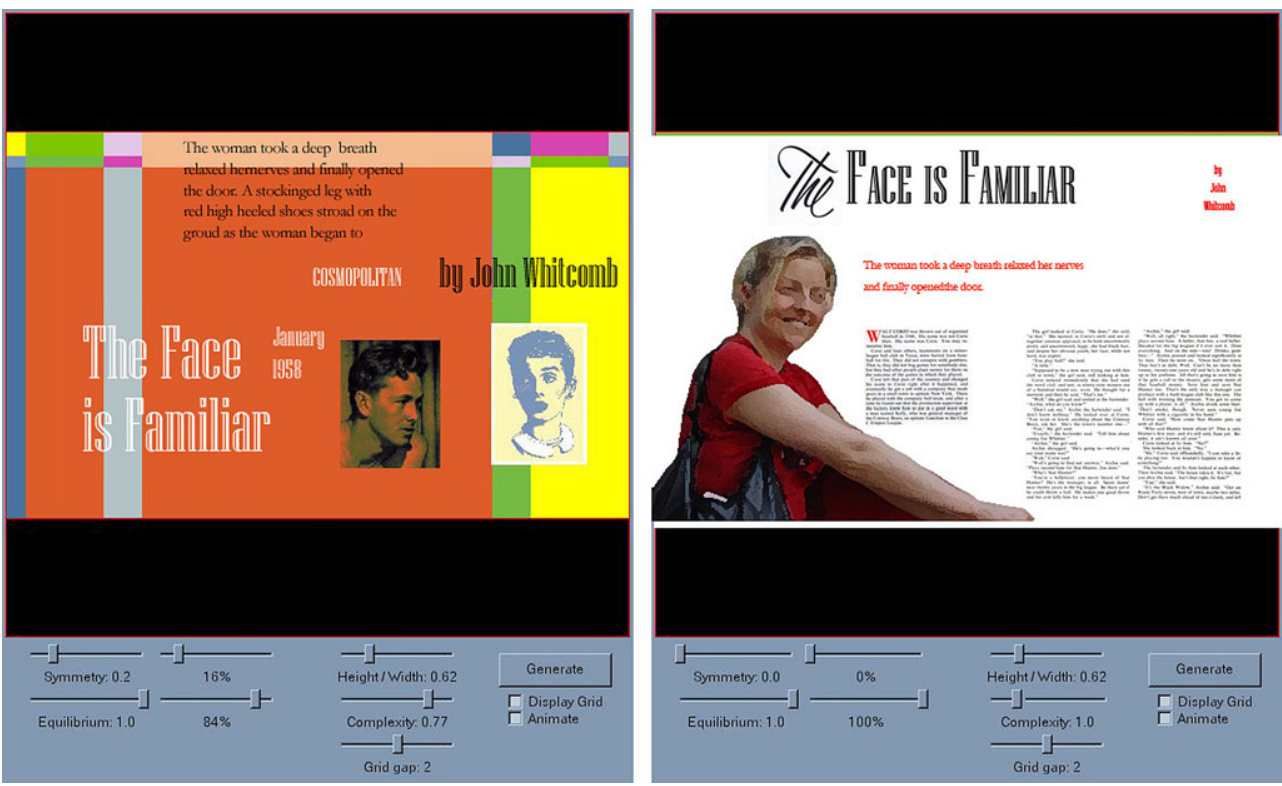

Figure 14 Two different solutions using background dominance in the layout 

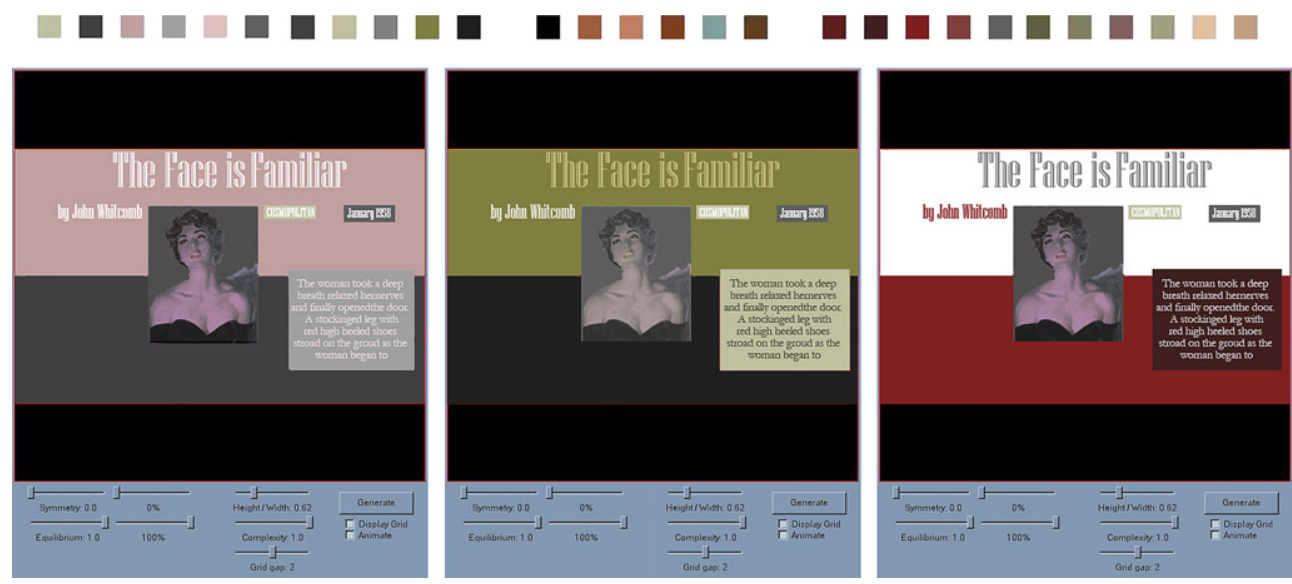

Figure 15 Colour solutions taken from predefined pallet library which is shown above the solutions

a geometric solution, and on the right an organic one based on 1950s genre graphics.

The SAI also holds information about text relationships as indicated in Figure 6. These are referenced to the overall size of the document page size. Having already established that the 1950s magazine genre we are looking at has a 10-20\% larger background ratio to foreground elements, we can adjust the size of the layout elements to suit. Firstly, the number of text elements is determined. In the example in Figure 17 there are five typographic elements; headline, author, magazine
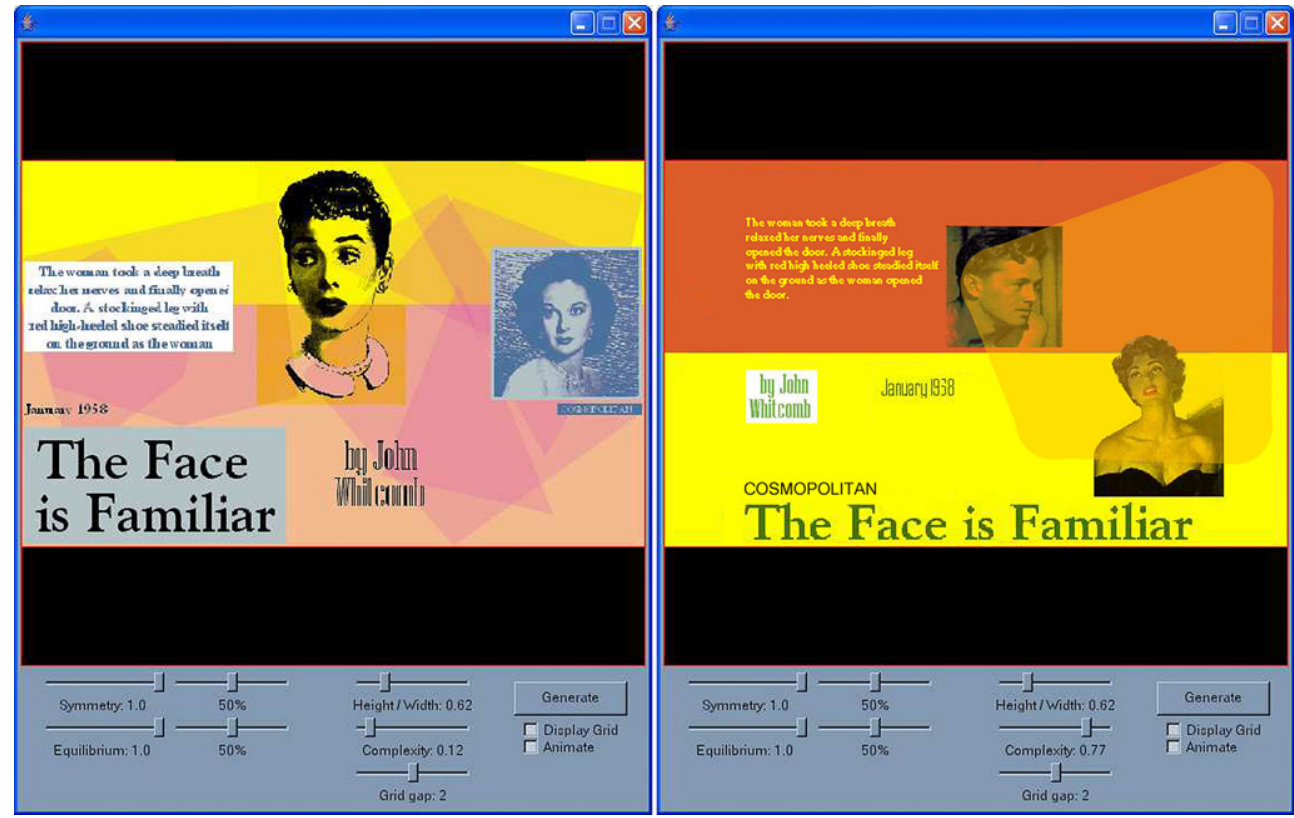

Figure 16 Geometric/Organic graphic objects

Style based automated graphic layouts 

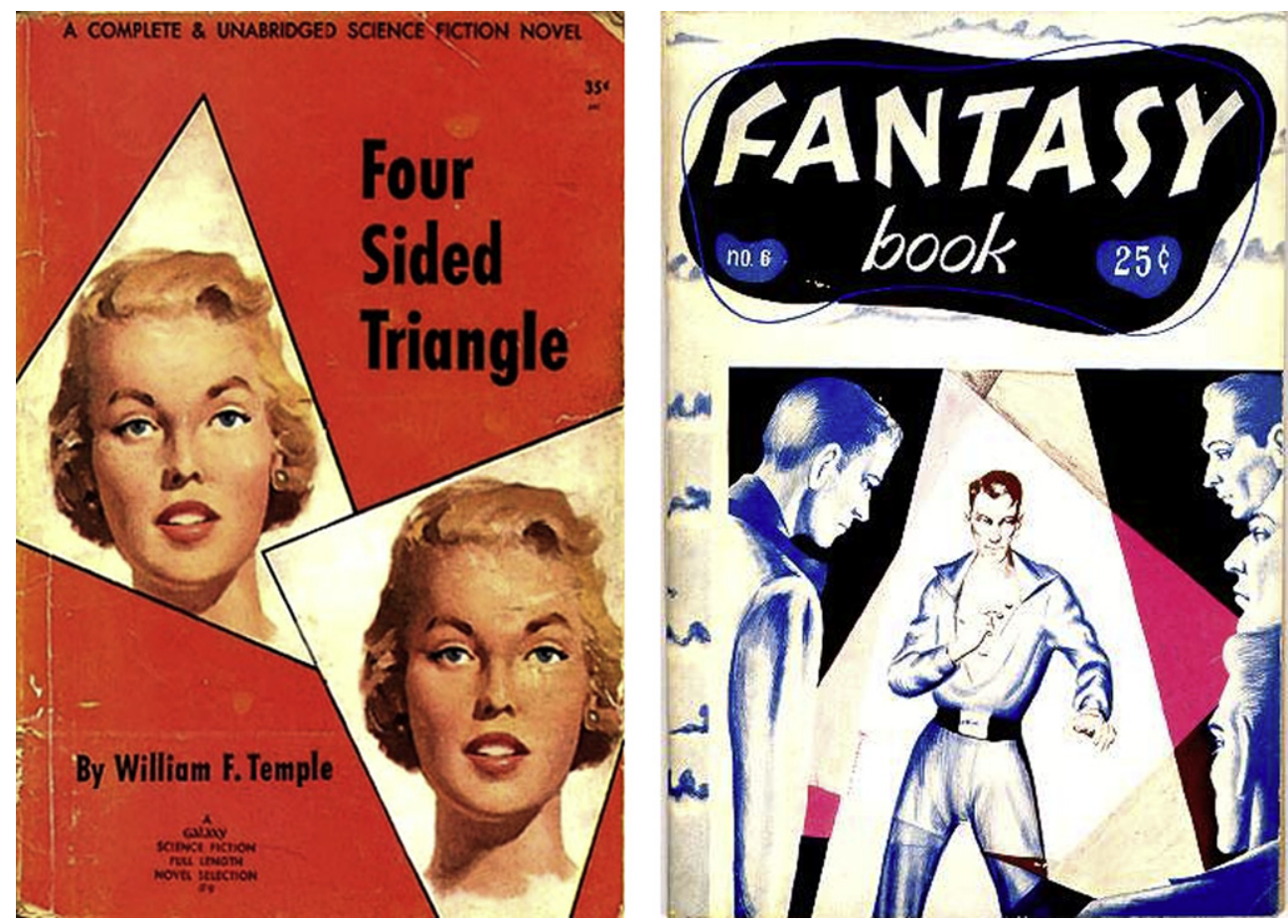

Figure 17 Examples of graphics generated using the Geometric/Organic parameter

name, date, and body text. This would be the saliency order for this particular genre. Saliency order is determined by tagging the text with the appropriate saliency value, the headline being given the value of 1 . This will then determine the corresponding typeface, point size, type style, tracking, leading and intertext relationship from a defined style parameter set. The size relationship is determined from the body text size. In this case the variation is set between 8 and 11 points. The algorithm randomly picks a point size for the body text between the two parameters and adjusts the other type sizes based on the saliency value.

The choice to typeface is determined from the type library which in this case takes a selection from a set of faces described as display and/or body text. These faces are tagged as appropriate for various style solutions.

So far we have discussed how the various elements in a layout are created and laid on to a grid after the calculation of how much negative space was appropriate for the required result. The issue of the overall relationship between the elements in terms of placement has not been addressed. This can best be explained by describing the process by which the final solution is arrived at. The program through a set of algorithms finds the elements to make up a design from various libraries which contain the fonts, text, pictures, graphic elements and colours. The program creates a random design from a selection 
of these elements and then determines the values for each of the parameters in the SAI. The design is then modified slightly by moving each element, modifying size and colour as well as type choice. If the new design has values which are close to the desired one it is kept, otherwise it is discarded and goes through the next iteration. The iterations go through several million cycles. The program terminates when one particular design remains the best solution. When the elements are placed they do so in a particular order. The first element to be placed is the body text, if there is body text, as this element often needs to be placed in aligned columns. This is followed by the illustrations/pictures/graphics, then by the headline and the other text elements as determined by the saliency order. As the elements populate the layout space their footprint is quarantined from any further placement, unless overlapping is permitted. The hierarchical order of text saliency allows placement in close proximity if space is available. Figure 18 illustrates this with three solutions, from hundreds of possibilities. Clearly some are more successful than others, and the relationship between the individual elements has a great effect on the correct reading of the style. For example, in Figure 18 the example on the left is an unusual solution for the 1950s genre, as the elements tend to float rather than attract each other as in the other examples. The placement of the headline at the bottom of the page, rather than in the top $50 \%$ of the page also changes the saliency compared to the other examples.

\section{Summary}

The SAI is capable of using preset parameters to save stylistic sets. The original objective of this project was to capture stylistic attributes in layouts and to be able to reproduce them in endless variations based on a set of design specifications. The generative engine which sorts these layout elements starts with random numbers as a starting point and then tries to apply the order logic of the SAI. The application of automated layout engines is of use in a number of

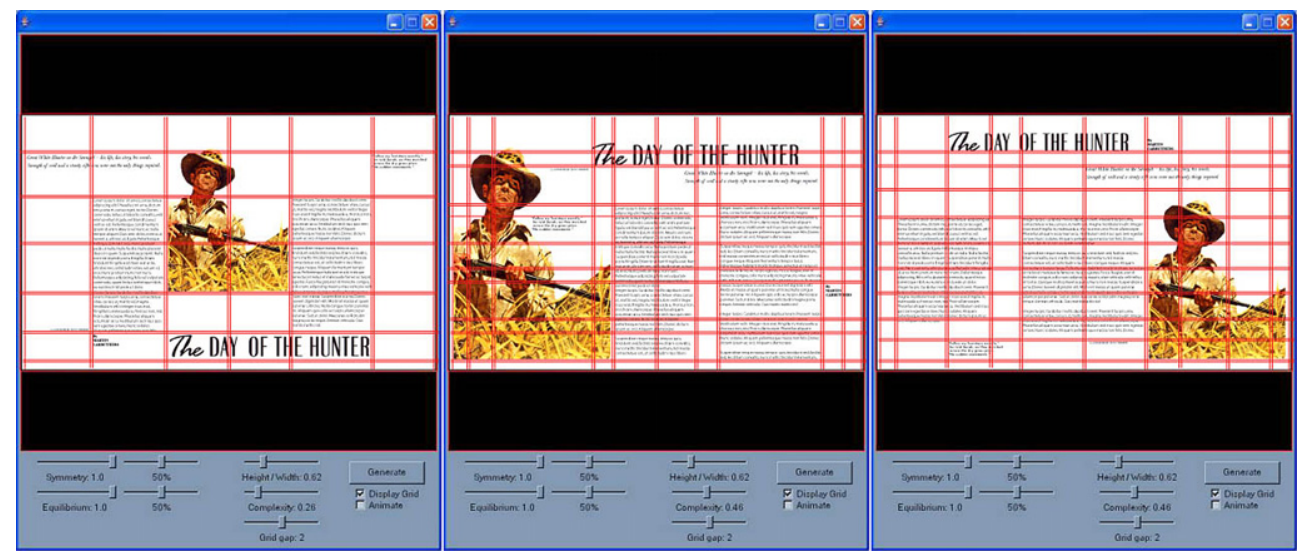

Figure 18 Examples of three generated layouts indicating the placement relationship of text

Style based automated graphic layouts 
layout applications such as continually updated complex web news and information sites. The intention is to see how feasible it would be for a designer to set a design specification and then to leave it to a set of algorithms to set variations on the layout using new sets of graphic and text elements.

The example given using the 1950s genre illustrates that use of the SAI is useful in producing layout designs which comply to a certain formula but offers a large number of possible solutions from which the designer can use as inspiration. The challenge is to develop a more robust version which can manipulate greater amounts of text with more refined parameters for placing these elements. The use of an SAI system has a number of benefits over the traditional use of layout templates which are often produced for non-designers. It offers unlimited possible solutions to a variety of user input variables. This is work in progress with further investigations aimed at refining the style logic and building appropriate libraries.

\section{References}

Birkhoff, G D (1993) Aesthetic measure, Harvard University Press, Cambridge Blackwell, L (2004) $20^{\text {th }}$ Century type Laurence King Publishing, London $\mathrm{p} 7$

Borning, A and Duisberg, R (1986) Constraint based tools for building user interfaces, ACM Transactions on Graphics Vol 5 No 4 pp 345-374

Cerf, M, Harel, J, Einhauser, W and Koch, C (2007) Predicting human gaze using low-level saliency combined with face detection Available from: $<$ http:// books.nips.cc/papers/files/nips20/NIPS2007_1074.pdf > [accessed online March 2009]

Cleveland, P (2004). The effect of technology on the development of magazine visual design style, $\mathrm{PhD}$ thesis, Griffith University, Brisbane

Itti, L and Koch, C (2001) Computational modelling of visual attention, Nature Reviews Neuroscience Vol 2 pp 1-11 March

Jacobs, C, Li, W, Schrier, E, Bargeron, D and Salesin, D (2004) Adaptive document layout, Communications of the ACM Vol 47 No 8 pp 60-66

Kadinsky, W (1979) Point and line to plane Dover, New York

Kay, P and Kempton, W (1984) What is the Sapir-Whorf hypothesis? American Anthropologist, New Series Vol 86 No 1 pp 65-79 Mar., 1984

Kress, G and Van Leeuwen, T (1996) Reading images: the grammar of visual design Routledge, London

Kress, G and Van Leeuwen, T (2001) Mulitmodal discourse - the modes and media of contemporary communication Arnold, London

Kress, G and Van Leeuwen, T (2002) Colour as a semiotic mode: notes for a grammar of colour, Visual Communication Vol 1 No 3 pp 343-369

Lee, D K, Itti, L, Koch, C and Braun, J (1999) Attention activates winner-take-all competition among visual filters, Nature Neuroscience Vol 2 No 4 pp 375-381

Lok, S, Feiner, S and Ngai, G (2004) Evaluation of visual balance for automated layout, IUI'04 Available from:<www.cs.columbia.edu/graphics/publications/ p101-lok.pdf $>$ pp 101-108

Myers, B A, McDaniel, R G and Kosbie, D S (1993) Marquise: creating computer user interfaces by demonstration, in Proceedings of INTERCHI'93, Human Factors in Computer Systems, pp 221-230

Scott, L M (1994) The Journal of Consumer Research Vol 21 No 2 pp 252-273 
Van Leeuwen, T (2006) Towards a semiotics of typography, Information Design Journal + Document Design Vol 14 No 2 pp 139-155

Vander Zanden, B and Myers, B A (1990) Automatic, look-and-feel independent dialogue creation of graphical user interfaces, in Proceedings of ACM CHI'90 Conference on Human Factors in Computer Systems

Yarbus, A L (1967) Eye movements and vision Plenum, New York 